\title{
NOUS NE SOMMES PAS DES CORPS GLORIEUX*
}

Sylvia Ostrowetsky**

A midi, chacun arrive avec lenteur près de la rampe qui mène à la salle à manger où les attendent, torchon blanc sur l'avant-bras, serveuses et serveurs.

Les handicapés, un membre dans le plâtre, sont sur des fauteuils roulants ou encore descendent la pente accrochées à leurs cannes, le regard légèrement inquiet. D'autres, plus âgé(e)s, maugréent en lisant le menu ou au contraire se réjouissent. La cohorte des obèses tente de se glisser entre les tables rondes recouvertes d'une nappe blanche ornée d'un bouquet d'œillets et s'installe avec difficulté sur des chaises toujours un peu trop étroites. Petit à petit le bruit s'installe depuis les fauteuils tirés, les cannes qui tombent, les pas pressés des serveuses, les voix des hôtes qui se saluent. Ils s'interrogent essentiellement sur leurs états respectifs de santé ou la qualité des plats: "Ca va?", "pas terrible, hein? aujourd'hui". Les jeunes s'interpellent volontiers d'un bout de la salle à l'autre en faisant de grands gestes, les dames plus âgées saluent avec politesse une personne d'une table voisine quand elles ont eu l'occasion de se connaître lors d'une activité commune organisée par la maison.

Mais les plus bruyants, ceux qui "rigolent pour un rien" aux dires des autres, ce sont, en général, les plus opulents. D’où leur vient ce goût de vivre qui s'étale avec insolence autant dans la voix qu'à travers les tressaillements du corps? Certaines cachent, derrière des vêtements amples, leurs rondeurs mais d'autres s'en moquent

\footnotetext{
* Presenté au Colloque international interdisiplinaire Corporéité, décorporéisation, vituralité: un état de la question du corps, Grenoble, 7, 8, 9 décembre 2000.

** Professeur des universités: Université de Picardie et CEFRESS-EDRESS.

Artigo recebido em 17 nov. 2004; aprovado em 15 dez. 2004.
} 
avec superbe ou du moins font semblant. Le plus gros, moustache à la gauloise mais doué d'une voix discrète, arbore des bretelles multicolores qui retiennent un pantalon qui n'en peut plus. La plus grosse est vêtue d'un collant bleu ciel de la couleur de ses yeux qui dessine avec exactitude tous les bourrelets d'une chair qui ne sait que déborder. Discrète, "gentille" - on aime beaucoup ce terme ici sa simplicité s'affiche avec ostentation. Elle n'a rien à cacher puisque le trop plein de graisse enfouit l'essentiel que trahit cependant des lèvres minces et pincées en permanence. A côté d'eux, car ils sont logiquement regroupés par tables de quatre au fond de la salle à manger, pas très loin des cuisines, une table de "régimes spéciaux". Ceux dont l'estomac ou l'intestin ne fonctionne pas bien et envers lesquels on a, à cause de leur maigreur, des attentions particulières comportant, entre autres, des plats supplémentaires qu'ils auront le droit de porter en vacillant jusqu'à leur chambre. Ils doivent théoriquement manger peu mais "fractionné". Autant les diététiciens s'échinent à leur conseiller des repas légers mais fréquents, autant les serveurs et servantes, s'imaginent qu'il faut les bourrer pour leur faire retrouver un profil normal. Une surtout empoisonne tout le monde de ses exigences incompréhensibles. Elle paraît sortir d'un camp et n'en veut jamais trop. Rien de cru, rien d'acide, les interdits sont implacables. Bousculé par ce qu'il leur semble un illogisme, le service a tacitement décidé de lui donner une portion normale en supplément de la portion diététique, si bien qu'elle est obligée de faire repartir régulièrement ses assiettes à moitié pleines. Elle a beau leur expliquer que sans estomac, cela passe avec difficulté, chacun insiste comme s'il parlait à une enfant pour qu'elle se nourrisse. Alors que chaque verre d'eau lui fait l'effet d'une pierre tombant sur l'intestin, on lui conseille de boire pour combler cette peau sèche et ridée qui ne peut s'enrichir de trop de graisses. La peau et les os... Avec un brin d'irritation certes, chacun a des attentions pour elle.

Après le repas, plusieurs - les obèses surtout - ramassent les croûtons de pains pour les poissons. Il y a, en effet, à l'autre bout du parc, un petit lac rempli de carpes. On ne les aperçoit pas d'emblée, mais il suffit de lancer un quignon pour en voir, une vingtaine 
quelquefois, se précipiter sur le morceau qui s'amollit progressivement et disparaît dans l'eau boueuse. Tandis qu'ils viennent de faire l'effort douloureux de manger sobrement, la goinfrerie animale compense, on peut le penser, une pulsion difficilement dominée. Une fois, une dame, qui vit en pavillon et "adore les bêtes", propose à notre femme en fil de l'accompagner et de jeter à son tour des morceaux à ce paquet d'écailles en ripaille. Le dégoût la possède.

Le jour du 14 juillet, un petit orchestre envahit la salle à manger que l'on a débarrassé de ses chaises et de ses tables. Germaine, la plus distinguée des obèses, invite le corps squelettique à danser. C'est l'enchantement. Le corps maigre vient s'emboîter dans le corps adipeux et les voilà tournoyant entre les autres danseurs, à l'endroit, à l'envers, dans une valse datée de leur jeunesse. Les hommes n'osent pas, ils sont trop gros mais les femmes, blondes, noires, beaucoup l'invitent et la font voltiger comme au manège.

Les plus âgées s'inquiètent. Elle est toute blanche, elle va se trouver mal. "Je la tiens !", rétorque Germaine en montrant ses bras vigoureux. N'empêche... L'une d'entre elles se lève et lui conseille de retourner dans sa chambre.

Le soir, la trop mince joue au rami avec les obèses qui avalent des bonbons au caramel qu'elle leur a apporté tandis qu'elle engouffre du chocolat. Elle perd à chaque partie sans que personne ne fasse la moindre remarque mais souvent, elles prennent la bonne carte à sa place parce qu'elle n'est pas assez rapide. De même, elles distribuent à sa place lorsque vient son tour afin d'éviter qu'elle ne se penche trop sur la table. Elle se laisse faire et éclate de rire quand, gagnante, l'une d'entre elles - il n'y a que les femmes qui y jouent - fait, vengeresse, un bras d'honneur au reste de la salle. Elle aime leur culot, leur façon de se moquer sans manière des hommes et de ne jamais se poser la question de leur féminité. Non qu'elles la mettent en doute, mais ce n'est ni dans la grâce des gestes, ni dans la délicatesse des mots qu'elle la situent. Dans le savoir-faire culinaire peut-être, dans les mèches décolorées, dans la responsabilité familiale? Une séduction sans apprêt. 
Le lendemain, une partie du groupe part en ballade. Les plus vigoureux s'efforcent de marcher au rythme de l'être filiforme qui n'a pas dit, quant à la résistance physique, son dernier mot. Elle tient bien le coup. On le lui dit avec sympathie.

Le dernier soir, on se retrouve dans la salle commune. Elle leur avoue qu'elle a commis quelques textes, dont l'un raconte en partie la dernière guerre. Avec sa trop lente dégaine et ses yeux un peu figés, sa maladie ressemble à la gamine fuyant la Gestapo son stigmate jaune sur le cœur.

Les obèses se protègent. Plus le corps est gros plus le moi, possédé par une pudeur infinie, rapetisse et se glisse dans les replis de la chair. C'est parce qu'elle est dépourvue de toute cuirasse adipeuse sans doute qu'ils entourent la trop mince silhouette. Du coup, on ne voit plus qu'elle avec son foulard multicolore et son ensemble gris "très classe".

Pour elle, les obèses sont l'excès d'un désir de grossir impossible. Pour les obèses, elle est la ligne d'horizon d'un désir de maigrir inatteignable. Souvent, elles lui disent en passant près d'elle, comme si elles n'étaient que de la "barbaque": " t'en veux un morceau?". Elle leur laisse le choix en leur montrant, en retour, la multitude d'endroits (seins, fesses) où ça manque.

Tant qu'elle n'était pas là, les obèses faisaient masse. Ils se tutoyaient, s'envoyaient des vannes comme des collégiens, se reconnaissaient. Chacun avait sa raison. Celui-là s'était séparé de sa femme, celle-là en avait marre d'être "cocue" et montrait à qui voulait la voir, la photo de son magnifique mari. L'autre ne disait rien mais le pli amer des lèvres parlait pour elle. Ils étaient ensemble parce qu'ils se ressemblaient, parce qu'ils savaient bien, eux, ce que recouvrait ces rires affichés, ces larges mouvements des bras... Passe-muraille, elle se glissait au milieu de cet antre maternel avec délice, certaine que chacun de ces corps l'accueillerait comme un fauteuil, la protégerait contre tout objet anguleux, toute agressivité maligne. Une joueuse de cartes, forte en belote, ne lui avait-elle pas reproché d'être 
trop lente. "Tu nous emmerdes, c'est la première fois qu'elle jouait" avait rétorqué Anne-Marie, et Michel avait approuvé.

Mais elle aussi leur donnait. D'abord, chacun avait bien repéré que sous l'argot les phrases étaient complètes. Elle "ne la ramenait pas", c'était essentiel mais quand il s'agissait de rédiger un mot, c'est à elle qu'on s'adressait. Pour les 25 ans de mariage de Germaine, elle rédigea un petit texte que tout le monde trouva "super" et qui fut signé par tous. Elle ressemblait un peu à une fleur fanée, à un oiseau blessé que l'on veut sauver alors que l'on se moque pas mal de ceux qui passent de vie à trépas sans qu'on les voit. Elle n'était ni charismatique, ni savante, ni douée d'une autorité naturelle: rien pour faire un chef. Elle ne faisait pas pitié non plus car tout le monde voyait bien que la vie tenait à ce corps quasi volatile. C'était autre chose; elle était un idéal qu'il ne fallait pas atteindre, un excès inversé qui avait acquis une délicatesse, une élégance qui leur était interdite. Le trop plein de substance des uns venait souligner l'existence à peine tracée de l'autre.

Fait de ressemblance et d'hilarité contagieuse, le groupe était envahissant. On ne voyait, on n'entendait qu' eux, les gros. La pointe du mépris était au bord du regard: ces gens pris dans la dépendance des bouches qui ne peuvent s'arrêter, des flaques. Avec son arrivée, leur grosseur fut encore plus visible et sa maigreur encore plus remarquable. Mais elle devint le fil d'Ariane, la métamorphose de l'entrelacs en labyrinthe. Sa seule présence organisa les échanges entre les formes rebondies et les creux, fournit à chacun une place à partir de quoi l'on pouvait se déplacer sans chavirer à chaque bosse. Plutôt, ce fut le fil qui fit cheminement, et le cheminement qui indiqua le centre à peine esquissé, dispersé, alternant avec des bouches toujours prêtes à se goinfrer et à se restreindre dans un tragique vaet-vient du remords gourmand. Pour les uns, il fallait résister à l'engouffrement. Pour elle, il fallait avaler, sentir la nourriture glisser difficilement à l'intérieur d'un magma désormais sans organe, passer jusqu'à la panse alambiquée des intestins. Souffrance des passages bloqués, du corps sectionné. 
Grâce à elle, ils surent l'extérieur de l'amaigrissement. Grâce à eux, elle sut ce qu' elle avait perdu: le rose de la chair, la jouissance de la surface. Ils lui donnaient leurs surplus graisseux; elle leur rendait, avec tendresse, la droiture des mots, la rigueur des formes.

A partir de cette rencontre étonnante tout fit sens, tout fut mobile et stable, tout se donna et se prit à la fois. Ils n'étaient plus une collectivité qui s'assemble parce qu'elle se ressemble. Entre eux, s'était glissée une épure qui donnait sens à leurs exagérations réciproques. Leurs connivences perdaient le poids de la banalité pour prendre une tournure quasi métaphysique.

Tout est question de culture n'est-ce-pas? Les orientaux aiment les parties bien charnues du corps tandis qu'ils détestent les maigres. De même Balzac décrit ainsi La cousine Bette: "Paysanne des Vosges dans toute l'extension du mot, maigre, brune, les cheveux d'un noir luisant, les sourcils épais et réunis par un bouquet, les bras longs et forts, les pieds épais, quelques verrues dans sa face longue et simiesque, tel est le portrait concis de cette vierge". Mais, dans un de ces premiers portraits romanesque de la laideur, il sait déjà que c'est affaire relative: " la chevelure noire, les beaux yeux durs, la rigidité des lignes du visage, la sécheresse calabraise du teint qui faisaient de la cousine Bette une figure de Giotto, et desquels une vraie parisienne eût tiré parti...". ${ }^{1}$ Dans Le martyre de l'obèse, Henri Béraud se plaint : "Rond, vous entendez, j'étais rond en voyant le jour et, depuis lors, on n'a cessé de me comparer à des objets renflés, à un pot à tabac, à un traversin, à Balzac". ${ }^{2}$

La laideur des obèses devient tout relative et se renverse presque avec l'arrivée de son négatif.

Comme ils sont beaux les cheveux blonds de ce Rubens, comme ses bras sont rassurants pour notre dame famélique. "Quelle élégance, elle peut tout mettre", dit Germaine en voyant filer notre Giacometti incarné!

Une norme quasi absente qui ne se mesure qu'à partir du trop, voilà, dirait Erving Goffman, les stigmatisés qui forment cet ensemble dysharmonieux mais organiquement construit et solide. 
Un regard collectif, dirais-je volontiers, qui fait de ce trop le signifiant majeur d'un renversement émotif. De l'euphorie de la minceur au dégoût de la maigreur, du goût de la rondeur au rejet des tissus marbrés par la cellulite.

Que les critères du beau changent aussi souvent qu'une mode, ce n'est pas ce banal propos - d'autant qu'il n'est pas si vrai - que nous reprenons ici, mais, dans ses replis, deux choses.

D'abord, non pas la norme en soi dont on sait combien elle peut changer comme on vient de le redire mais, comme le dit goffman encore: sa hantise. J'ajouterai ensuite, que cette hantise, nous conjoint, fait lien, encore plus fort peut-être que les Valeurs puisées dans la transcendance dont on nous dit - on veut bien le croire - qu'elles sont en déshérence.

Sans un mot, le regard nous lie les uns aux autres à travers un jugement dit Kant quasi sans appel malgré son extrême versatilité: le jugement esthétique.

Ce groupe improbable aura réussi à faire société à partir de ses insuffisances et de ses surplus.

Passé le temps du séjour en maison de convalescence, ils se reverront. Ils souffrent, ils vivent, ils ont plus à partager que leurs ressemblances qui possèdent désormais la puissance socialisatrice d'un concept.

\section{Notes}

1 Paris: Gallimard, 1972.p. 59.

2 Paris: Albin Michel, 1986. p. 13. Balzac a-t-il fait de Bette une maigre pour atténuer sa propre laideur? C'est une question de mode bien sûr, de relativité culturelle du goût, dont il s'agit. 
